\title{
Genomic instability and myelodysplasia with monosomy 7 consequent to EVI1 activation after gene therapy for chronic granulomatous disease
}

\author{
Stefan Stein ${ }^{1,15}$, Marion G Ott ${ }^{2,15}$, Stephan Schultze-Strasser ${ }^{1}$, Anna Jauch $^{3}$, Barbara Burwinkel ${ }^{4,5}$, Andrea Kinner ${ }^{1}$, \\ Manfred Schmidt ${ }^{6}$, Alwin Krämer ${ }^{7}$, Joachim Schwäble ${ }^{2}$, Hanno Glimm ${ }^{6}$, Ulrike Koehl ${ }^{8}$, Carolin Preiss ${ }^{1}$, \\ Claudia Ball ${ }^{6}$, Hans Martin ${ }^{2}$, Gudrun Göhring ${ }^{9}$, Kerstin Schwarzwaelder ${ }^{6}$, Wolf-Karsten Hofmann ${ }^{10}$, \\ Kadin Karakaya $^{7}$, Sandrine Tchatchou ${ }^{4,5}$, Rongxi Yang ${ }^{4,5}$, Petra Reinecke ${ }^{11}$, Klaus Kühlcke ${ }^{12}$, Brigitte Schlegelberger ${ }^{9}$, \\ Adrian J Thrasher ${ }^{13}$, Dieter Hoelzer ${ }^{2,15}$, Reinhard Seger ${ }^{14,15}$, Christof von Kalle ${ }^{6,15}$ \& Manuel Grez ${ }^{1,15}$ \\ Gene-modified autologous hematopoietic stem cells (HSC) can provide ample clinical benefits to subjects suffering from X-linked \\ chronic granulomatous disease (X-CGD), a rare inherited immunodeficiency characterized by recurrent, often life-threatening \\ bacterial and fungal infections. Here we report on the molecular and cellular events observed in two young adults with X-CGD \\ treated by gene therapy in 2004. After the initial resolution of bacterial and fungal infections, both subjects showed silencing \\ of transgene expression due to methylation of the viral promoter, and myelodysplasia with monosomy 7 as a result of insertional \\ activation of ecotropic viral integration site 1 (EVII). One subject died from overwhelming sepsis 27 months after gene therapy, \\ whereas a second subject underwent an allogeneic HSC transplantation. Our data show that forced overexpression of EVI1 \\ in human cells disrupts normal centrosome duplication, linking EVI1 activation to the development of genomic instability, \\ monosomy 7 and clonal progression toward myelodysplasia.
}

Treatment of primary immunodeficiencies with gene-modified autologous stem cells may offer considerable benefits over allogeneic cell transplantation, particularly when human leukocyte antigen (HLA)matched donors are not available. Phase $1 / 2$ clinical trials aimed at the correction of X-linked severe combined immunodeficiency (SCID-X1) and adenosine deaminase-deficient SCID (ADA-SCID) using gammaretroviral vectors have successfully demonstrated reconstitution of immunity and correction of metabolic toxicities ${ }^{1-5}$. However, the occurrence of leukemias as a consequence of retroviral integration observed in five SCID-X1 cases has illustrated the risk associated with inadvertent proto-oncogene activation ${ }^{6-9}$.

We applied gene therapy to treat $\mathrm{X}$-linked chronic granulomatous disease (X-CGD), a rare inherited immunodeficiency that is characterized by recurrent severe bacterial and fungal infections due to a functional defect in the microbe-killing activity of phagocytic neutrophils ${ }^{10-13}$. Two young adults with X-CGD were treated with autologous gene-modified cells in combination with nonmyeloablative conditioning. Successful engraftment of gene-transduced cells resulted in the eradication of pre-existing life-threatening bacterial and fungal infections. However, insertional activation of MDS1-EVI1 and the genes encoding PR domain-containing protein 16 (PRDM16) and SET-binding protein 1 (SETBP1) triggered a threefold to fivefold increase in the number of gene-transduced cells in the peripheral blood of both subjects ${ }^{14}$, leading to oligoclonal hematopoiesis, monosomy 7 and the development of a myelodysplastic syndrome (MDS).

\section{RESULTS}

Clinical findings

After gene therapy, subject 1 remained well and clinically stable for almost 18 months, during which time we discontinued antimicrobial prophylaxis. However, after a routine checkup at month 15, we noted declining white blood cell counts (platelets, $45,000 \mu \mathrm{l}^{-1}$; neutrophils, 1,600 $\mathrm{ll}^{-1}$; lymphocytes, 1,200 $\mu \mathrm{l}^{-1}$ ) (Fig. 1a). Bone marrow biopsies taken at months 15 and 18 showed declining cellularity with left-shifted hematopoiesis and an increased number of macrophages, but no signs of myelodysplasia or malignancy (Supplementary Fig. 1a).

\footnotetext{
${ }^{1}$ Institute for Biomedical Research, Georg-Speyer-Haus, Frankfurt, Germany. ${ }^{2}$ Department of Hematology/Oncology, University Medical School, Frankfurt, Germany. ${ }^{3}$ Institute of Human Genetics, University Hospital Heidelberg, Heidelberg, Germany. ${ }^{4}$ Molecular Epidemiology Group, German Cancer Research Center, Heidelberg, Germany. ${ }^{5}$ University Women's Clinic, Division Molecular Biology of Breast Cancer, Heidelberg, Germany. ${ }^{6}$ Department of Translational Oncology, National Center for Tumor Diseases and German Cancer Research Center, Heidelberg, Germany. ${ }^{7}$ Clinical Cooperation Unit Molecular Hematology/Oncology, German Cancer Research Center and Department of Internal Medicine V, University of Heidelberg, Heidelberg, Germany. ${ }^{7}$ Pediatric Hematology, Oncology and Hemostaseology, University Medical School, Frankfurt, Germany. ${ }^{8}$ Department of Cell and Molecular Pathology, Hannover Medical School, Hannover, Germany. ${ }^{9}$ Department of Hematology, Oncology and Transfusion Medicine, Charité, Campus Benjamin Franklin, Berlin, Germany. ${ }^{10}$ Institute of Pathology, Heinrich-Heine University, Düsseldorf, Germany. ${ }^{11}$ EUFETS AG, Idar-Oberstein, Germany. ${ }^{12}$ Centre for Immunodeficiency, UCL Institute of Child Health, and Great Ormond Street Hospital for Children NHS Trust London, UK. ${ }^{13}$ Division of Immunology/Hematology, University Children's Hospital Zurich, Zurich, Switzerland. ${ }^{15}$ These authors contributed equally to this work. Correspondence should be addressed to M.G. (grez@em.uni-frankfurt.de).
}

Received 19 March 2009; accepted 18 December 2009; published online 24 January 2010; doi:10.1038/nm.2088 
Figure 1 Hematopoietic reconstitution, gene marking and superoxide production in subjects 1 and 2 after gene therapy. (a,b) White blood cell (WBC), platelet, lymphocyte, hemoglobin $(\mathrm{Hb})$ and absolute neutrophil counts (ANC) after gene therapy are shown for subjects 1 (a) and 2 (b). (c,d) Quantification of genemodified cells and GP91-PHOX mRNA levels in peripheral blood granulocytes and reconstitution of superoxide activity after gene therapy for subjects 1 (c) and 2 (d).

The subject received daily granulocyte colonystimulating factor (G-CSF) support ( $5 \mu \mathrm{g}$ per $\mathrm{kg}$ body weight per day) from months 18 to 20 and months 24 to 26 , as well as multiple red blood and platelet transfusions. Following a dental abscess and a febrile episode requiring antibiotic and antimycotic treatment, subject 1 was noted to have extensive splenomegaly and underwent splenectomy at month 25 to avoid spontaneous rupture. Histopathological examination of the spleen revealed extramedullary hematopoiesis and siderosis in the red pulp, without signs of dysplasia, and increased infiltration with activated macrophages showing hemophagocytosis. After a transient improvement, at month 26, he developed a pseudomembranous colitis with perforation and underwent colectomy. He died 27 months after gene therapy of multiorgan failure owing to septic shock. The postmortem pathology is described in detail in the Supplementary Notes. Retrospectively, the occurrence of cytopenia and the presence of uniformly vacuolated monocytic cells in the peripheral blood at month 27, together with the finding of monosomy 7 (see below), indicates that subject 1 developed MDS as consequence of gene therapy, probably with transition to acute myeloid leukemia (AML) with organ infiltration at the time of death.

Routine clinical and laboratory examinations of subject 2 up to 22 months after gene therapy documented a stable clinical status, with no severe infections and no alterations in peripheral blood counts or bone marrow morphology. However, 28 months after gene therapy, he developed mild pancytopenia (macrocytic, hyperchromic anemia; hemoglobin, $11.8 \mathrm{~g} \mathrm{dl}^{-1}$; platelets, 125,000 $\mathrm{ul}^{-1}$; decreasing neutrophil counts) (Fig. 1b). Serial routine bone marrow biopsies revealed normal cellularity and cytogenetics until month 33, when we first noted mild hypocellularity (approximately $70 \%$ of normal cell concentrations), without dysplasia or blasts (Supplementary Fig. 1d). By month 43, a bone marrow aspirate revealed severe hypocellularity, increased erythropoiesis and multilineage dysplasia with less than 5\% blast cells (Supplementary Fig. 1e,f), consistent with refractory cytopenia with multilineage dysplasia (RCMD) according to the World Health Organization (WHO) criteria. At month 45, subject 2 underwent allogeneic stem cell transplantation from a fully matched, unrelated donor. For details, see the Supplementary Notes and Supplementary Figure 2.

Gene marking and NADPH oxidase activity in transduced cells As a result of EVI1- and MDS1-EVI1-triggered clonal expansion ${ }^{14}$, the percentages of gene-transduced peripheral blood neutrophils remained between $50 \%$ and $65 \%$ until month 26 and month 36 for subjects 1 and 2, respectively (Fig. 1c,d). Thereafter, we observed a decrease in gene marking in peripheral blood granulocytes of subject 2, with $30 \%$ gene-transduced cells present at 40 months after
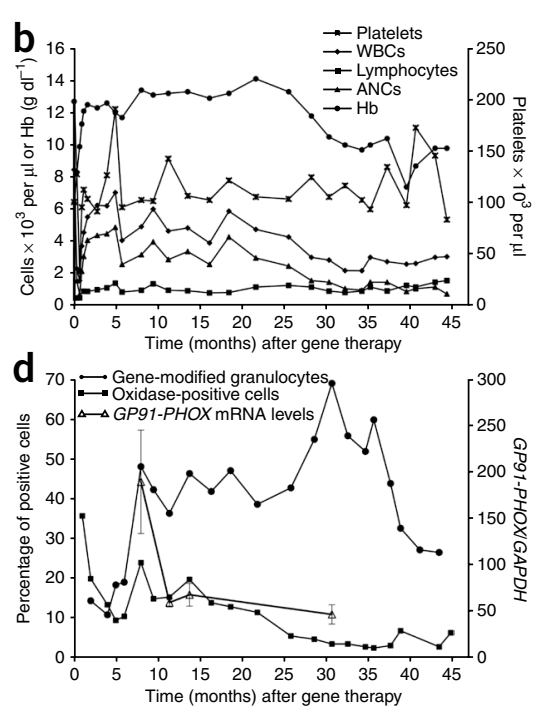

transplantation. Gene marking in bone marrow mononuclear cells (BM MNCs) of subject 2 remained constant, between $40 \%$ and $50 \%$, whereas gene marking in lymphocytes remained stable at $10 \%-15 \%$ in B cells and less than 3\% in T cells (Supplementary Fig. 3a,b). Despite high numbers of gene-marked cells, the number of oxidase-positive granulocytes progressively decreased from month 9 and month 15 for subjects 1 and 2, respectively, and correlated with a concomitant decrease in the amount of GP91-PHOX transcript (encoding gp91 phox) in peripheral blood granulocytes (Fig. 1c,d). We used bisulfite sequencing to analyze vector methylation in samples from subjects 1 and 2 and found that increased methylation at a CpG island within the promoter region of the viral long terminal repeat (LTR) correlated with the lack of GP91-PHOX expression and superoxide production observed in the peripheral blood cells of both subjects (Fig. 2a and Supplementary Fig. 4). Notably, we detected no methylation at $\mathrm{CpG}$ dinucleotides within the enhancer region of the LTR, suggesting a selective pressure for this epigenetic fingerprint.

\section{Clonal distribution of gene-marked cells}

The highly polyclonal population of transduced cells observed shortly after transplantation ${ }^{14}$ progressively became clonally restricted and dominated by clones containing retroviral integrations in the MDS1-EVI1 gene locus (Fig. 2b). In peripheral blood samples of subject 1 , we detected a single dominant clone containing the integration $75916 \mathrm{~A} 08$, initially at month 4 at a frequency of $3 \%$ within total gene-marked hematopoiesis. Its contribution increased steadily, reaching 35\% at month 11 (Fig. 2c). Methylation analysis of this clone revealed a nonmethylated LTR (Supplementary Fig. 5), linking clone 75916A08 to the restored superoxide production observed in granulocytes from this subject during the first 10-18 months after gene therapy. From month 24 onward the clonal repertoire was dominated by clone $76776 \mathrm{G} 11$, which contained a fully methylated promoter region and accounted for $>90 \%$ of the genemarked hematopoiesis at month 26 (Fig. 2c and Supplementary Fig. 5). As both dominant clones contained integrations in the MDS1 gene locus, increased expression of EVI1 or secondary mutations may have contributed to the dominance of clone $76776 \mathrm{G} 11$ over other clones with similar integrations.

We observed a similar shift toward oligoclonality in peripheral blood cells of subject 2. Two MDS1-EVI1 clones, 87429F02 and 87429A02, dominated gene-marked hematopoiesis between 
Figure 2 Proviral DNA methylation, clonal distribution of gene-marked cells and MDS1-EVI1 activation in blood samples of subjects 1 and 2. (a) Sequential analysis of $\mathrm{CpG}$ methylation at the proviral LTR promoter region in peripheral blood (PB) samples of subjects 1 and 2 after gene therapy. (b) Analysis of retroviral insertion sites by linear amplification-mediated PCR (LAM-PCR) in PB samples of subjects 1 and 2 (months 26 and 44, bone marrow samples). The identity of the dominant LAM-PCR bands and the sampling month are indicated. IC, internal control; C, negative control; M, marker DNA. (c) Longitudinal tracking of dominant clones in PB samples of subjects 1 (above) and 2 (below) after gene therapy. Depicted is the retrieval frequency of individual clones obtained by LAM-PCR and pyrosequencing analysis. (d) RT-PCR analysis for GP91-PHOX, MDS1-EVI1 and EVI1 transcripts in samples from subjects 1 (above) and 2 (below) after gene therapy. Above, MDS1-EVII and EVII transcripts in PBMCs (lanes 2, 4 and 9-10), granulocytes (lanes 3, 5, 6 and 8) or total PB (lane 7) from subject 1 at different time points. Lane 1 , control a
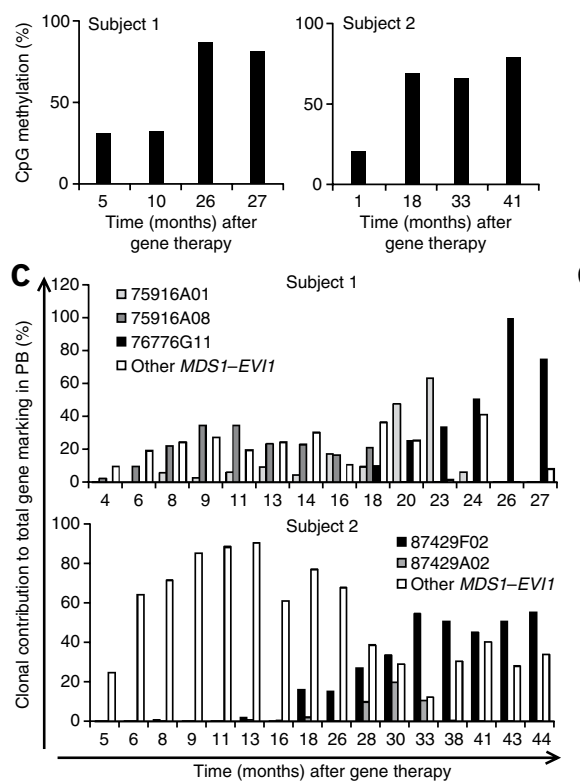

b Subject 1

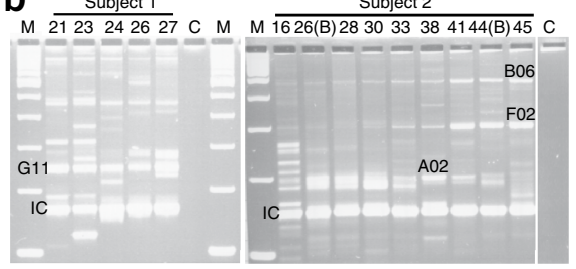

d
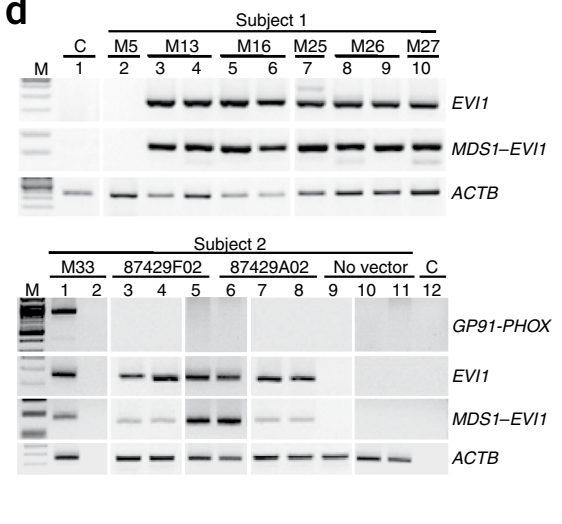

\section{Subject 2}

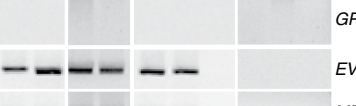
GP91-PHOX EVI1 MDS1-EVI1 ACTB

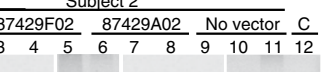
before gene therapy; lane 5, fluorescence-activated cell sorting (FACS)-sorted gp91 ${ }^{\text {phox }}$-positive granulocytes; lane 6, negative fraction of the same sample. Below, GP91-PHOX, MDS1-EVI1 and EVII transcripts in granulocytes of subject 2 (lane 1) 33 months after gene therapy, in samples derived from isolated bone marrow colonies with the integrations 87429F02 (lanes 3-5) or 87429A02 (lanes 6-8) and in colonies derived from nontransduced progenitors (lane 9-11). Lane 2, control without reverse transcriptase; lane 12, water control (C). M, marker DNA. ACTB, actin control gene.

months 28 and 33 (Fig. 2b,c), but at later time points, we detected only clone $87429 \mathrm{~F} 02$. This clone showed extensive methylation at the promoter region of the viral LTR, which was already detectable 5 months after transplantation (Supplementary Fig. 5).

In both subjects 1 and 2, we detected MDS1-EVI1 and EVI1 transcripts in peripheral blood mononuclear cells (PBMCs) and granulocytes after gene therapy, but they were absent from samples taken before gene therapy, clearly indicating that transcriptional activation of the MDS1-EVI1 gene locus was retrovirus dependent (Fig. 2d). In both subjects the MDS1-EVI1 transcript contained the second exon of the MDS1 gene spliced to the second exon of the EVI1 gene, generating a fusion transcript identical to that found in leukemias with 3 q26 rearrangements ${ }^{15}$, and the EVI1 transcripts contained exon 1 b. In subject 2, we also detected EVI1 transcripts and MDS1-EVI1 fusion transcripts in bone marrow-derived colonies containing either the 87429F02 or the 87429A02 integration (Fig. 2d, below). EVI1 expression increased with time in both subjects, and EVI1 transcript levels in the dominant clones 76776G11 (subject 1) and 87429F02 (subject 2) were comparable to those found in a human cell line with EVI1 rearrangement ${ }^{16}$. In contrast, MDS1-EVI1 expression contributed less than $1 \%$ to the total level of EVI1 transcript in the dominant clones (Supplementary Fig. 6).

\section{Clonal dominance is associated with monosomy 7}

Using a retrospective chromosome copy number variation (CNV) analysis based on single-nucleotide polymorphism (SNP) arrays, we identified a series of chromosomal regions that were amplified in both subjects, mainly at months 26 and 27 for subject 1 and months 35 and 38 for subject 2 (Supplementary Table 1). From month 21 onward, we identified a loss of one chromosome 7 in the PBMCs of subject 1 (Fig. 3a). The frequency of cells containing a single chromosome 7 increased up to $80 \%$ at month 27 , a time point at which clone $76776 \mathrm{G} 11$ dominated gene-marked hematopoiesis $(>75 \%$ contribution to gene marking). Results obtained by interphase fluorescence in situ
Figure 3 Monosomy 7 in dominant clones of subjects 1 and 2. (a-c) Detection of monosomy 7 by SNP arrays and interphase FISH in cells from subject 1. (a) SNP arrays on peripheral blood mononuclear samples of subject 1 at different time points show increasing numbers of cells containing a single chromosome 7 . The log2 signal ratio of each SNP (red dots) on the Affymetrix GeneChip 100K array set (month 21, $50 \mathrm{~K}$ array), the local averaged $\log 2$ signal ratio ( $k=10$ SNPs, blue curve) and the inferred copy number according to the hidden Markov model (dashed lines) for chromosome 7 are shown. The ideogram of chromosome 7 is shown below the averaged log2 signal ratio curve (blue line). The green bars below the ideogram represent heterozygous signals. (b,c) Interphase FISH analysis for chromosome 7 (7q31, SpectrumOrange LSI D7S486 and 7 centromere, SpectrumGreen, CEP7) of mononuclear cells obtained either from bone marrow at month 13 (b) or peripheral blood of subject 1 at month 27 after gene therapy (c). (d) Interphase FISH analysis for chromosome 7 of bone marrow MNCs obtained from subject 233 months after gene therapy. (e,f) Monosomy 7 was most predominant in cells isolated from bone marrow-derived colonies containing integration $87429 \mathrm{FO} 2$ (e) but was not detected in nontransduced cells (f). Scale bar, $10 \mu \mathrm{m}$.

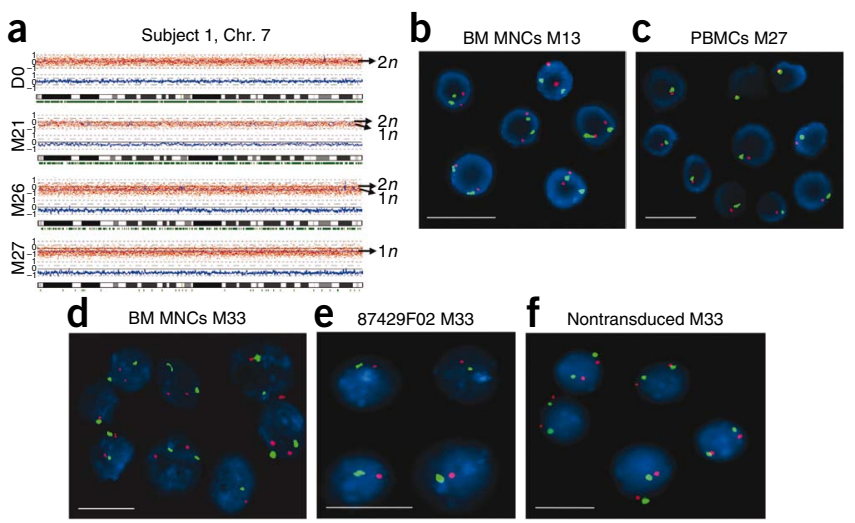


Figure 4 Genomic instability in MDS1-EVI1-expressing cells. (a,b) Immunofluorescence staining for phosphorylated $\mathrm{H} 2 \mathrm{AX}(\gamma-\mathrm{H} 2 \mathrm{AX})$ on either total peripheral blood (month 4) or PBMCs (month 27) from subject 1 (a) or BM MNCs from subject 2 (b) collected at different time points during gene therapy. Cells taken before gene therapy were analyzed as a control (subject 1, total PB; subject 2, marrow MNCs). For each time point, foci of 250-600 individual nuclei (157 for subject 2, M26) were quantified on confocal picture stacks using the 'object counter 3D' plug-in of the MBF bundle for the ImageJ software (http://rsbweb.nih.gov/ij/). Shown are maximum intensity projections of picture stacks. The $\gamma-\mathrm{H} 2 \mathrm{AX}$ staining is pseudocolored in red, and nuclei were counterstained with TOT03 (blue). The mean number of foci per cell is indicated below. $(\mathbf{c}, \mathbf{d})$ Graphical display of the distribution of cells (percentage) containing a distinct number of foci for subjects 1 (c) and 2 (d). Scale bar, $5 \mu \mathrm{m}$.

hybridization (I-FISH) were consistent with these findings. At month 27 we detected monosomy 7 in almost all PBMCs, whereas at month 13 we found no chromosomal abnormalities BM MNCs (Fig. 3b,c).

In subject 2 , there were no changes in chromosomal copy number in BM MNCs for the first 33 months, as analyzed by I-FISH. However, at month 33 we detected monosomy 7 in BM MNCs at a frequency of about $25 \%$, as shown by I-FISH and metaphase chromosome analysis (Fig. 3d and Supplementary Fig. 7b). Thereafter, the percentage of monosomy 7 cells in subject 2 increased to $50 \%$ at month 45 . Analysis of single hematopoietic colonies derived from bone marrow samples from subject 2 showed that monosomy 7 was linked to clone 87429F02 (Fig. 3e) and was not detectable in nontransduced cells (Fig. 3f). Notably, metaphase (M)-FISH analysis of interleukin-2 (IL-2)-expanded T cells of subject 2 from months 31 and 33 did not show any chromosomal rearrangements (Supplementary Fig. 7c), suggesting that monosomy 7 originated in at least one, probably myeloid, progenitor cell in which the MDS1-EVI1 gene locus was transcriptionally activated by retroviral insertion.

The occurrence of monosomy 7 together with the clinical findings for both subjects was consistent with a diagnosis of MDS. In line with this observation, a genome-wide methylation analysis of 807 cancer-related
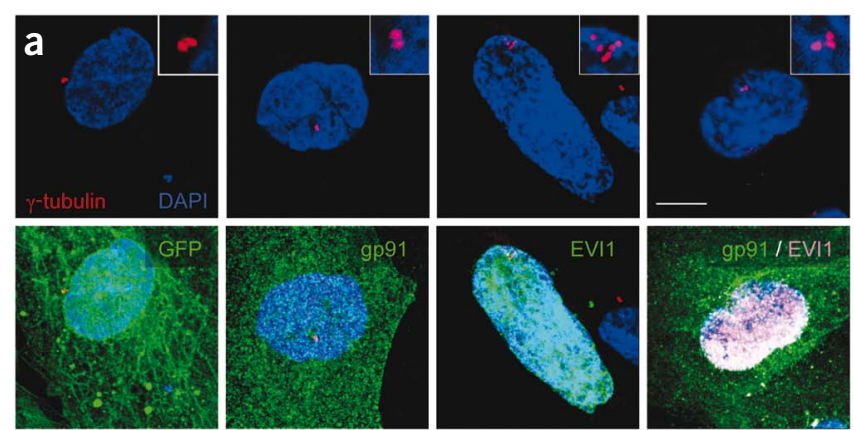

b

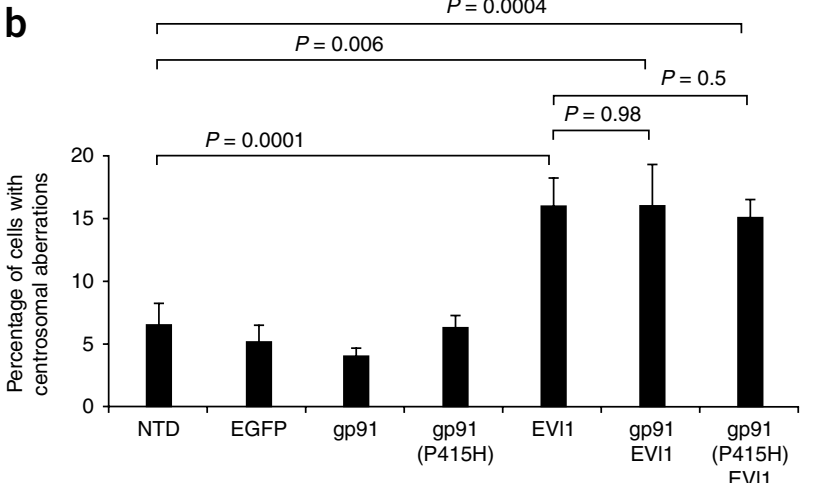

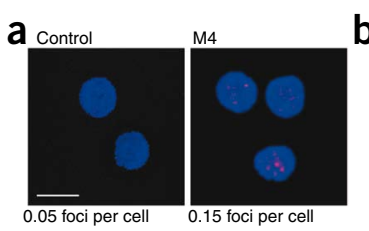
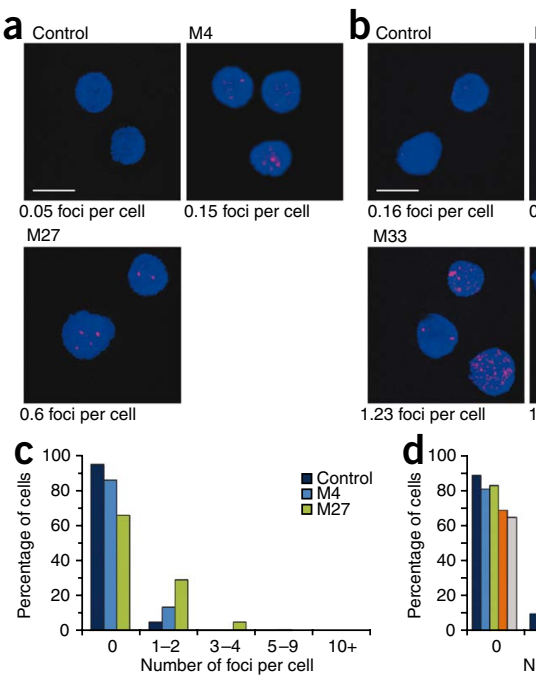

M33
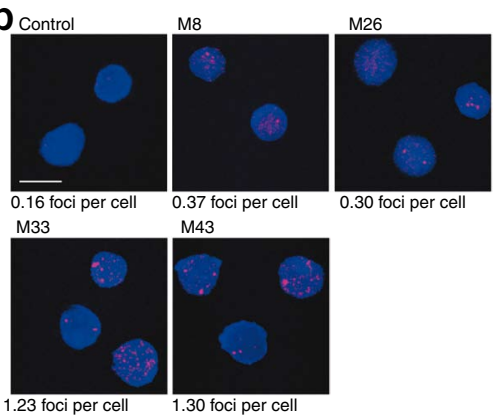

M43
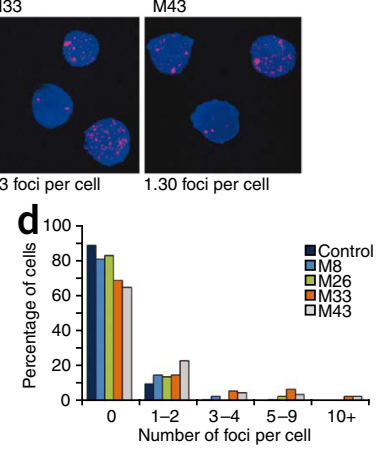

genes revealed a DNA methylation pattern characteristic of patients with MDS and AML ${ }^{17,18}$ (Supplementary Tables 2 and 3). Among others, the gene encoding $\mathrm{p} 15^{\mathrm{INK} 4 \mathrm{~B}}, C D K N 2 B$, whose frequent methylation in individuals with MDS or AML is associated with deletions of genes on chromosome $7 \mathrm{q}^{19}$, was hypermethylated in peripheral blood samples from both subjects. We found that $C D K N 2 B$ methylation correlated well with the appearance of monosomy 7-containing cells in the peripheral blood of subjects 1 and 2 (Supplementary Fig. 8).

Increased genomic instability in MDS1-EVI1-expressing cells Phosphorylation of histone $\mathrm{H} 2 \mathrm{AX}$ is one of the earliest events detectable after induction of DNA double-strand breaks (DSBs) ${ }^{20-22}$. We detected an 8-fold to 12-fold increase in the number of foci per cell with phosphorylated $\mathrm{H} 2 \mathrm{AX}(\gamma-\mathrm{H} 2 \mathrm{AX})$ in PBMCs of subject 1 at month 27 and BM MNCs of subject 2 at month 43 . Whereas before gene therapy almost no $\gamma$-H2AX foci were detectable in peripheral blood samples from subject 1, at month 4 we detected DSBs in $~ 14 \%$ of the cells, with a mean value of 0.15 foci per cell (Fig. 4). At this time point, the total MDS1-EVI1 clonal contribution to genemodified cells was $13.2 \%$. At 27 months after gene therapy, when gene-marked hematopoiesis was dominated by the monosomy 7 clone 76776 G11 (Fig. 2c), the concentration of DSBs in PBMCs increased to a mean value of 0.6 foci per cell, with $34 \%$ of the cells having one or more $\gamma$-H2AX foci per cell (Fig. 4a,c). In subject 2, the percentage

Figure 5 EVI1 induces centrosomal aberrations in human cells. Human diploid BJ fibroblasts were stably transduced with vectors expressing either eGFP, gp91 $1^{\text {phox }}$ or gp91 ${ }^{\text {phox }}(\mathrm{P} 415 \mathrm{H})$, a catalytically inactive mutant of gp91 ${ }^{\text {phox }}$. EVII was transiently expressed in nontransduced or in gp91 ${ }^{\text {phox }}$-expressing and gp91 ${ }^{\text {phox }}$ (P415H)-expressing fibroblasts. Cells were stained for $\gamma$-tubulin to analyze for centrosomal abnormalities. (a) EGFP-, gp91 ${ }^{\text {phox }}$ - or EVI1-expressing cells were stained with specific antibodies to EGFP, EVI1 or gp91 ${ }^{\text {phox }}$ (green). Double-transduced cells expressing gp91 ${ }^{\text {phox }}$ and EVI1 were stained with specific antibodies to gp91 ${ }^{\text {phox }}$ (green) and EVI1 (lilac). Centrosomes were visualized by $\gamma$-tubulin staining and are pseudocolored in red. Nuclei were counterstained with DAPI or TOPRO-3 (blue). Shown are maximum intensity projections of confocal picture stacks. Enlarged areas with stained centrosomes are shown in the right upper corner. (b) Percentage of cells with aberrant numbers $(>2)$ of centrosomes. Only cells expressing the transgenes were analyzed. Data were obtained from at least three sets of 100 analyzed cells. Statistical significance was determined by Student's $t$-test. Scale bar, $10 \mu \mathrm{m}$. 
of marrow-derived MNCs containing $\geq 3$ foci per cell increased from $1 \%$ before gene therapy to $16 \%$ at month 33 , with a mean value of 0.16 foci per cell and 1.23 foci per cell, respectively (Fig. $\mathbf{4 b}, \mathbf{d}$ ).

As genomic instability has been linked to centrosomal aberrations ${ }^{23-25}$, we transduced human diploid BJ fibroblasts with EVI1 expression constructs to determine whether overexpression of EVI1 induces centrosomal amplification. Compared to BJ fibroblasts transduced with a vector expressing enhanced green fluorescent protein (EGFP), cells overexpressing EVI1 showed an increased frequency of centrosomal aberrations, as determined by increased numbers of centrosomes in almost $16 \%$ of the EVI1-expressing cells (Fig. 5). Similarly, overexpression of MDS1-EVI1 in BJ fibroblasts led to a twofold to threefold increase in centrosome numbers, suggesting equal functions for EVI1 and MDS1-EVI1 in this regard (Supplementary Fig. 9).

In contrast, the number of centrosomes in fibroblasts stably expressing gp91 ${ }^{\text {phox }}$ or its catalytic inactive form, gp91 ${ }^{\text {phox }}(\mathrm{P} 415 \mathrm{H})^{26} \mathrm{did}$ not differ from that found in nontransduced cells or in cells expressing EGFP (Fig. 5). We conclude that the insertional activation of EVI1 was the dominant factor leading to centrosomal aberrations and genomic instability in our patients that resulted in the development of MDS with monosomy 7 .

\section{DISCUSSION}

Gene therapy provided clear clinical benefit in this clinical trial ${ }^{14}$, with both subjects initially recovering from life-threatening bacterial and fungal infections. However, long-term benefit was compromised by vector inactivation, resulting in a lack of microbicidal activity in gene-transduced phagocytic cells. Although vector inactivation has been reported in cell lines, embryonic and mouse hematopoietic stem cells $^{27,28}$, methylation of the vector promoter has not been reported in clinical studies so far. In other immunodeficiencies, powerful positive-selection pressures may have obscured this phenomenon, as nonexpressing progenitors would not support further survival and differentiation. However, a partial methylation of the spleen focusforming virus (SFFV) LTR has been observed in a clinical trial of gene therapy for ADA-SCID (ref. 4 and A. J. Thrasher, personal communication), suggesting that the SFFV LTR is prone to methylation in human hematopoietic cells.

In X-CGD, expression of gp91 ${ }^{\text {phox }}$ is not necessary for cell survival, and gp $91^{\text {phox }}$ is not known to confer a proliferative advantage to myeloid progenitors. A direct role for the therapeutic gp $91^{\text {phox }}$ protein in the clonal proliferation observed in our study is unlikely because gp91 ${ }^{\text {phox }}$ expression decreased with time in both subjects owing to silencing of the LTR promoter. Although initially there was a good correlation between clonal expansion and GP91-PHOX expression in the peripheral blood of subject 1 , and to a lesser extent also in subject 2, within the first months after gene therapy, we eventually detected methylation-dependent silencing of GP91-PHOX expression in most, but not all, of the clones analyzed (Supplementary Fig. 5). Methylation of the viral promoter seems to correlate with global methylation at the site of integration ${ }^{29}$, suggesting that the epigenetic events occurring at the vector LTR in our trial reflected epigenetic marks at the site of integration. The lack of methylation at the enhancer region of the LTR may reflect a selective pressure for a vector-dependent transactivation of EVI1 and MDS1-EVI1, as clonal expansion was linked to the continuous expression of these proto-oncogenes.

Gene-transduced hematopoiesis in both subjects was dominated by clones containing retroviral insertions within the MDS1-EVI1 gene locus. This locus represents one of the most common targets of retrovirus integration observed in vitro and in vivo ${ }^{30}$. In animal models, retrovirus-mediated transcriptional activation of Evil has been associated with extensive proliferation and self-renewal of hematopoietic progenitor cells, increased engraftment potential and clonal dominance ${ }^{31,32}$. In mouse hematopoiesis, the insertional activation of Evil can also lead to leukemia of myeloid or lymphoid origin ${ }^{33,34}$, whereas ectopic expression of Evil in bone marrow cells induces a fatal MDS after an initial period of myeloproliferation ${ }^{35,36}$. However, chromosomal aberrations as a consequence of Evil overexpression have not been reported in any in vitro or in vivo study to date ${ }^{30,34}$.

In humans, transcriptional activation of EVI1 is often associated with MDS and $\mathrm{AML}^{37-39}$. In MDS, rearrangements involving $3 \mathrm{q} 26$ (EVI1) can be found either as the only cytogenetic aberration, or associated with additional chromosomal abnormalities, most often monosomy 7 (refs. 40-43). Neither the exact link between 3q26 rearrangements and loss of one chromosome 7 , nor the sequential order of events leading to MDS, has been clarified. Gene marking of individual hematopoietic clones by retroviral insertion provides a basis to dissect the order and mechanism of the molecular and cellular events leading to malignancy. In the evolution of leukemia in the gene therapy studies for SCID-X1, oncogene activation through insertional mutagenesis was associated with the accumulation of additional genetic lesions ${ }^{8,9}$. In our study, we observed profound clonal expansions driven by the insertional activation of EVI1, MDS1-EVI1 and PRDM16, a homolog of MDS1-EVI1, in multiple clones of both subjects 5 months after gene therapy. We detected no abnormalities in blood counts, bone marrow morphology or karyotype during this period, indicating that this insertional event was probably the driving event for progression toward clonal dominance.

In addition to the role of EVI1 and MDS1-EVI1 in development, transcriptional regulation, self-renewal and cell cycle progression ${ }^{44-47}$, EVI1 has been shown to interact with proteins involved in the chromatin-remodeling process, including BRG1, SUV39H1, G9a and MBD3B, and may thereby influence proper centrosome replication, chromatin assembly and genomic stability ${ }^{48-52}$. During mitosis, centrosomes function as spindle poles, directing the formation of bipolar spindles, a process essential for accurate chromosome segregation ${ }^{53}$. Centrosome amplification may lead to chromosomal instability and has been frequently observed in both solid tumors and hematological malignancies, including myelodysplastic syndromes and $\mathrm{AML}^{23,24}$. In line with these observations, our analysis indicates profound genomic instability in EVI1- and MDS1-EVI1-expressing clones. At month 27, PBMCs of subject 1 showed a 12-fold increase in the number of $\gamma$-H2AX foci per cell, compared to control cells. Similarly, we observed a 1.9-fold to 8.1-fold increase in the number of foci per cell in samples taken from subject 2 .

The increase in DNA DSBs preceded the onset of myelodysplasia and, together with increasing clonal dominance, was highly predictive of abnormal hematopoiesis. Genomic instability was linked to the aberrant expression of EVI1 and MDS1-EVI1, as overexpression of either gene in human diploid fibroblasts caused a twofold to threefold increase in abnormal centrosome numbers in transduced cells. We speculate that this effect could be mediated by the binding of EVI1 to G9a, a H3K9-specific histone methyltransferase. EVI1-G9a complexes have reduced methytransferase activity, and downregulation of G9a activity has been linked to centrosome disruption and chromosome instability in cancer cells ${ }^{50,52}$.

Although we observed increased EVI1 and MDS1-EVI1 expression in the bulk population of expanded hematopoietic clones, two clones, one in each subject, dominated gene-marked hematopoiesis at later time points. Both clones, 76776G11 (subject 1) and 87429F02 (subject 2), 
expressed EVI1 at levels similar to those found in leukemic cells containing EVI1 rearrangements, and both lost one copy of chromosome 7. In contrast, MDS1-EVI1 transcript levels were at least 100 -fold lower than those of EVI1. It is therefore tempting to speculate that deletion of one chromosome 7 occurred in clones expressing EVI1 at high levels. This hypothesis is supported by the observations made for subject 2. At month 11, the total contribution of clones containing MDS1-EVI1 integrations to gene-marked hematopoiesis in peripheral blood was $87 \%$, but total EVI1 expression was low. At month 37, the contribution of the monosomy 7-containing clone to gene marking was almost $50 \%$, whereas EVI1 expression levels were equal to or higher than that found in a leukemic cell line with inv(3).

Alternatively, high EVI1 expression levels may have been triggered by haploinsufficiency of EVI1 transcriptional regulators on chromosome 7. In this context, it is noteworthy that the myeloid/lymphoid or mixed-lineage leukemia 5 gene (MLL5), a trithorax homolog with a crucial role in adult hematopoiesis and a postulated role in chromatin remodeling and in the control of cellular growth, was recently mapped to $7 \mathrm{q} 22$, a region that is frequently deleted in $\mathrm{AML}^{54-57}$.

A contribution of gp91 $9{ }^{\text {phox }}$ expression to aberrant hematopoiesis is unlikely, as was observed promoter methylation in the monosomy 7-containing clones as early as 1 month after gene therapy in the dominant clone $76776 \mathrm{G} 11$ and 5 months after transplantation in the dominant clone 87429F02, much earlier than the onset of dysplasia and monosomy 7 . Also, forced expression of gp $91^{\text {phox }}$ in human diploid fibroblasts did not induce alterations in centrosome numbers above those found in mock-transduced cells or in cells expressing a catalytically inactive form of gp91 ${ }^{\text {phox }}$. In agreement with this, we have not detected increased concentrations of reactive oxygen species in gp91 ${ }^{\text {phox }}$-expressing cells. Similarly, we believe that the administration of G-CSF did not influence disease progression, as disease development and outcome was identical in both subjects, but only subject 1 received G-CSF (further details in the Supplementary Notes).

Despite the severe adverse effects observed in our trial, the initial eradication of preexisting life-threatening bacterial and fungal infections and other clinical benefits experienced by both subjects for more than 2 years after reinfusion of their autologous gene-modified cells are clear evidence that gene therapy can be an effective strategy for the long-term correction of CGD. Improved vector designs should enable the safe and effective application of gene therapy for the long-term correction of CGD and other inherited disorders, as demonstrated recently ${ }^{58}$.

\section{METHODS}

Methods and any associated references are available in the online version of the paper at http://www.nature.com/naturemedicine/.

\section{Note: Supplementary information is available on the Nature Medicine website.}

\section{ACKNOWLEDGMENTS}

We are indebted to K. Bleuel and S. Wehner for contributions and advice during the study; to H. Holtgreve-Grez and B. Schoell for I-FISH and M-FISH analysis, respectively; to $\mathrm{H}$. Konrad Müller-Hermelink for reference pathology; to B. Staehle for quantitative PCR; to M.C. Dinauer (Indiana University School of Medicine) for plasmid gp91 $1^{\text {phox }}(\mathrm{P} 415 \mathrm{H})$ and to H. Kunkel, E. Rudolf, A. Dillmann, R. Kramer, B. Moreno, I. Vogler and L. Chen-Wichmann for assistance during this work. This work was supported by the Bundesministerium für Bildung und Forschung (grant 01GU0507, TP6b), the Chronic Granulomatous Disorder Research Trust, London (grant J4G/04B/GT), the Georg-Speyer-Haus (T300131), the EU (VIth Framework Program, CONSERT), the Research Priority Program 1230 from the Deutsche Forschungsgemeinschaft and, in part, by a grant of the Tumorzentrum
Heidelberg/Mannheim to A.J. A.J.T. is also supported by the Wellcome Trust and the Department of Health (HTH/011/025/004). A. Krämer is supported by the Deutsche Krebshilfe (grant 108560). The Georg-Speyer-Haus is supported by the Bundesministerium für Gesundheit and the Hessisches Ministerium für Wissenschaft und Kunst.

\section{AUTHOR CONTRIBUTIONS}

S.S., M.G.O. and M.G. conceived, organized and supervised the study; S.S., A. Kinner, S.S.-S., C.P. and K. Kühlcke performed experiments; B.B., S.T. and R.Y. performed the SNP and methylation arrays and analyzed data; A. Kinner,

A. Krämer, C.B., K. Karakaya. and H.G. generated and analyzed data on genomic instability; A.J. performed the I-FISH and M-FISH analysis; S.S.-S. performed the CpG methylation studies; M.G.O., J.S., H.M., G.G., W.-K.H., P.R., B.S., U.K., R.S. and D.H. were responsible for patient care, analyzed materials from the subjects and provided data on these samples; M.S., K.S. and C.v.K. provided data on retroviral integrations sites; A.J.T., R.S., U.K., M.S., C.v.K. and M.G. prepared and wrote the manuscript.

\section{COMPETING INTERESTS STATEMENT}

The authors declare no competing financial interests.

Published online at http://www.nature.com/naturemedicine/.

Reprints and permissions information is available online at http://npg.nature.com/ reprintsandpermissions/.

1. Cavazzana-Calvo, M. et al. Gene therapy of human severe combined immunodeficiency (SCID)-X1 disease. Science 288, 669-672 (2000).

2. Aiuti, A. et al. Correction of ADA-SCID by stem cell gene therapy combined with nonmyeloablative conditioning. Science 296, 2410-2413 (2002).

3. Gaspar, H.B. et al. Gene therapy of X-linked severe combined immunodeficiency by use of a pseudotyped gammaretroviral vector. Lancet 364, 2181-2187 (2004).

4. Gaspar, H.B. et al. Successful reconstitution of immunity in ADA-SCID by stem cell gene therapy following cessation of PEG-ADA and use of mild preconditioning. Mol. Ther. 14, 505-513 (2006).

5. Aiuti, A. et al. Gene therapy for immunodeficiency due to adenosine deaminase deficiency. N. Engl. J. Med. 360, 447-458 (2009).

6. Hacein-Bey-Abina, S. et al. LMO2-associated clonal T cell proliferation in two patients after gene therapy for SCID-X1. Science 302, 415-419 (2003).

7. Hacein-Bey-Abina, S. et al. A serious adverse event after successful gene therapy for X-linked severe combined immunodeficiency. N. Engl. J. Med. 348, 255-256 (2003).

8. Howe, S.J. et al. Insertional mutagenesis combined with acquired somatic mutations causes leukemogenesis following gene therapy of SCID-X1 patients. J. Clin. Invest. 118, 3143-3150 (2008).

9. Hacein-Bey-Abina, S. et al. Insertional oncogenesis in 4 patients after retrovirusmediated gene therapy of SCID-X1. J. Clin. Invest. 118, 3132-3142 (2008).

10. Dinauer, M.C. The respiratory burst oxidase and the molecular genetics of chronic granulomatous disease. Crit. Rev. Clin. Lab. Sci. 30, 329-369 (1993).

11. Roos, D. The genetic basis of chronic granulomatous disease. Immunol. Rev. 138 121-157 (1994).

12. Segal, A.W. The NADPH oxidase and chronic granulomatous disease. Mol. Med. Today 2, 129-135 (1996).

13. Malech, H.L. \& Hickstein, D.D. Genetics, biology and clinical management of myeloid cell primary immune deficiencies: chronic granulomatous disease and leukocyte adhesion deficiency. Curr. Opin. Hematol. 14, 29-36 (2007).

14. Ott, M.G. et al. Correction of X-linked chronic granulomatous disease by gene therapy, augmented by insertional activation of MDS1-EVI1, PRDM16 or SETBP1. Nat. Med. 12, 401-409 (2006).

15. Fears, S. et al. Intergenic splicing of MDS1 and EVI1 occurs in normal tissues as well as in myeloid leukemia and produces a new member of the PR domain family. Proc. Natl. Acad. Sci. USA 93, 1642-1647 (1996).

16. Hamaguchi, H. et al. Establishment of a novel human myeloid leukaemia cell line (HNT-34) with $\mathrm{t}(3 ; 3)(\mathrm{q} 21 ; \mathrm{q} 26), \mathrm{t}(9 ; 22)(\mathrm{q} 34 ; \mathrm{q} 11)$ and the expression of EVI1 gene, P210 and P190 BCR/ABL chimaeric transcripts from a patient with AML after MDS with 3q21q26 syndrome. Br. J. Haematol. 98, 399-407 (1997).

17. Claus, R. \& Lubbert, M. Epigenetic targets in hematopoietic malignancies. Oncogene 22, 6489-6496 (2003).

18. Nolte, F. \& Hofmann, W.K. Myelodysplastic syndromes: molecular pathogenesis and genomic changes. Ann. Hematol. 87, 777-795 (2008).

19. Christiansen, D.H., Andersen, M.K. \& Pedersen-Bjergaard, J. Methylation of p15INK4B is common, is associated with deletion of genes on chromosome arm $7 \mathrm{q}$ and predicts a poor prognosis in therapy-related myelodysplasia and acute myeloid leukemia. Leukemia 17, 1813-1819 (2003).

20. Rogakou, E.P., Pilch, D.R., Orr, A.H., Ivanova, V.S. \& Bonner, W.M. DNA doublestranded breaks induce histone $\mathrm{H} 2 \mathrm{AX}$ phosphorylation on serine 139. J. Biol. Chem. 273, 5858-5868 (1998).

21. Khanna, K.K. \& Jackson, S.P. DNA double-strand breaks: signaling, repair and the cancer connection. Nat. Genet. 27, 247-254 (2001). 
22. Fernandez-Capetillo, O., Celeste, A. \& Nussenzweig, A. Focusing on foci: H2AX and the recruitment of DNA-damage response factors. Cell Cycle 2, 426-427 (2003).

23. Kearns, W.G., Yamaguchi, H., Young, N.S. \& Liu, J.M. Centrosome amplification and aneuploidy in bone marrow failure patients. Genes Chromosomes Cancer 40, 329-333 (2004).

24. Neben, K., Giesecke, C., Schweizer, S., Ho, A.D. \& Kramer, A Centrosome aberrations in acute myeloid leukemia are correlated with cytogenetic risk profile. Blood 101, 289-291 (2003).

25. Ganem, N.J., Godinho, S.A. \& Pellman, D. A mechanism linking extra centrosomes to chromosomal instability. Nature 460, 278-282 (2009).

26. Dinauer, M.C., Curnutte, J.T., Rosen, H. \& Orkin, S.H. A missense mutation in the neutrophil cytochrome b heavy chain in cytochrome-positive X-linked chronic granulomatous disease. J. Clin. Invest. 84, 2012-2016 (1989).

27. Wang, L., Robbins, P.B., Carbonaro, D.A. \& Kohn, D.B. High-resolution analysis of cytosine methylation in the $5^{\prime}$ long terminal repeat of retroviral vectors. Hum. Gene Ther. 9, 2321-2330 (1998).

28. Frank, O. et al. Tumor cells escape suicide gene therapy by genetic and epigenetic instability. Blood 104, 3543-3549 (2004).

29. Burns, W.R., Zheng, Z., Rosenberg, S.A. \& Morgan, R.A. Lack of specific gammaretroviral vector long terminal repeat promoter silencing in patients receiving genetically engineered lymphocytes and activation upon lymphocyte restimulation. Blood 114, 2888-2899 (2009).

30. Metais, J.Y. \& Dunbar, C.E. The MDS1-EVI1 gene complex as a retrovirus integration site: impact on behavior of hematopoietic cells and implications for gene therapy. Mol. Ther. 16, 439-449 (2008).

31. Du, Y., Jenkins, N.A. \& Copeland, N.G. Insertional mutagenesis identifies genes that promote the immortalization of primary bone marrow progenitor cells. Blood 106, 3932-3939 (2005)

32. Calmels, B. et al. Recurrent retroviral vector integration at the MDS1-EVII locus in non-human primate hematopoietic cells. Blood 106, 2530-2533 (2005).

33. Li, Z. et al. Murine leukemia induced by retroviral gene marking. Science 296 , 497 (2002).

34. Modlich, U. et al. Leukemia induction after a single retroviral vector insertion in Evil or Prdm16. Leukemia 22, 1519-1528 (2008).

35. Buonamici, S. et al. EVI1 induces myelodysplastic syndrome in mice. J. Clin. Invest. 114, 713-719 (2004).

36. Du, Y., Spence, S.E., Jenkins, N.A. \& Copeland, N.G. Cooperating cancer-gene identification through oncogenic-retrovirus-induced insertional mutagenesis. Blood 106, 2498-2505 (2005).

37. Nucifora, G. The EVI1 gene in myeloid leukemia. Leukemia 11, 2022-2031 (1997).

38. Dreyfus, F. et al. Expression of the Evi-1 gene in myelodysplastic syndromes. Leukemia 9, 203-205 (1995).

39. Barjesteh van Waalwijk van Doorn-Khosrovani, S et al. High EVII expression predicts poor survival in acute myeloid leukemia: a study of 319 de novo AML patients. Blood 101, 837-845 (2003).
40. Charrin, C. et al. Structural rearrangements of chromosome 3 in 57 patients with acute myeloid leukemia: clinical, hematological and cytogenetic features. Hematol. J. 3, 21-31 (2002)

41. Lin, P., Medeiros, L.J., Yin, C.C. \& Abruzzo, L.V. Translocation (3;8)(q26;q24): a recurrent chromosomal abnormality in myelodysplastic syndrome and acute myeloid leukemia. Cancer Genet. Cytogenet. 166, 82-85 (2006).

42. Stevens-Kroef, M. et al. Translocation $\mathrm{t}(2 ; 3)(\mathrm{p} 15-23 ; \mathrm{q} 26-27)$ in myeloid malignancies: report of 21 new cases, clinical, cytogenetic and molecular genetic features. Leukemia 18, 1108-1114 (2004).

43. Trubia, M. et al. Characterization of a recurrent translocation $t(2 ; 3)(p 15-22 ; q 26)$ occurring in acute myeloid leukaemia. Leukemia 20, 48-54 (2006).

44. Laricchia-Robbio, L. \& Nucifora, G. Significant increase of self-renewal in hematopoietic cells after forced expression of EVI1. Blood Cells Mol. Dis. 40, 141-147 (2007).

45. Perkins, A.S., Mercer, J.A., Jenkins, N.A. \& Copeland, N.G. Patterns of Evi-1 expression in embryonic and adult tissues suggest that Evi-1 plays an important regulatory role in mouse development. Development 111, 479-487 (1991).

46. Kustikova, O.S. et al. Retroviral vector insertion sites associated with dominant hematopoietic clones mark "stemness" pathways. Blood 109, 1897-1907 (2007).

47. Senyuk, V. et al. Repression of RUNX1 activity by EVI1: a new role of EVI1 in leukemogenesis. Cancer Res. 67, 5658-5666 (2007).

48. Chi, Y., Senyuk, V., Chakraborty, S. \& Nucifora, G. EVI1 promotes cell proliferation by interacting with BRG1 and blocking the repression of BRG1 on E2F1 activity. J. Biol. Chem. 278, 49806-49811 (2003).

49. Cattaneo, F. \& Nucifora, G. EVI1 recruits the histone methyltransferase SUV39H1 for transcription repression. J. Cell. Biochem. 105, 344-352 (2008).

50. Spensberger, D. \& Delwel, R. A novel interaction between the proto-oncogene Evi1 and histone methyltransferases, SUV39H1 and G9a. FEBS Lett. 582, 2761-2767 (2008).

51. Spensberger, D. et al. Myeloid transforming protein Evi1 interacts with methyl-CpG binding domain protein 3 and inhibits in vitro histone deacetylation by $\mathrm{Mbd} 3 / \mathrm{Mi}-2 /$ NuRD. Biochemistry 47, 6418-6426 (2008).

52. Kondo, Y. et al. Downregulation of histone H3 lysine 9 methyltransferase G9a induces centrosome disruption and chromosome instability in cancer cells. PLoS One 3, e2037 (2008).

53. Krämer, A., Neben, K. \& Ho, A.D. Centrosome replication, genomic instability and cancer. Leukemia 16, 767-775 (2002).

54. Zhang, Y. et al. MLL5 contributes to hematopoietic stem cell fitness and homeostasis. Blood 113, 1455-1463 (2009).

55. Heuser, M. et al. Loss of MLL5 results in pleiotropic hematopoietic defects, reduced neutrophil immune function, and extreme sensitivity to DNA demethylation. Blood 113, 1432-1443 (2009).

56. Madan, V. et al. Impaired function of primitive hematopoietic cells in mice lacking the mixed-lineage-leukemia homolog MLL5. Blood 113, 1444-1454 (2009).

57. Sebastian, S. et al. MLL5, a trithorax homolog, indirectly regulates H3K4 methylation, represses cyclin A2 expression, and promotes myogenic differentiation. Proc. Natl. Acad. Sci. USA 106, 4719-4724 (2009).

58. Cartier, N. et al. Hematopoietic stem cell gene therapy with a lentiviral vector in X-linked adrenoleukodystrophy. Science 326, 818-823 (2009). 


\section{ONLINE METHODS}

Methods. Subject characteristics, gene therapy treatment and the follow-up methodology used including quantitative PCR, immunophenotyping, analysis of transgene function by superoxide production, LAM-PCR ${ }^{59}$ and clonal tracking have been described ${ }^{14}$ (ClinicalTrials.gov identifier: NCT00564759). Additional methods can be found in the Supplementary Methods. The cellular composition of the subject samples analyzed in Figures 1-4 is described in the Supplementary Notes. All experiments involving human subjects were approved by the local Ethics Review Board of the University of Frankfurt Medical School and the Commission for Somatic Gene Therapy of the German Medical Council.

59. Schmidt, M. et al. High-resolution insertion-site analysis by linear amplificationmediated PCR (LAM-PCR). Nat. Methods 4, 1051-1057 (2007). 